\title{
Über die Veränderung in Psychiatrie und Psychotherapie
}

\author{
Berthold Rothschild (Zürich)
}

\begin{abstract}
Zusammenfassung: Anlässlich einer Neukonzeption und der damit verbundenen Neueröffnung der früheren Psychiatrischen Klinik Oberwil/Franziskusheim am Zugersee wurde der Autor gebeten, eine Standortbestimmung zur heutigen stationären Psychiatrie vorzutragen und aufderen Veränderungen bis zur Gegenwart einzugehen. Der Autor weist auf einige wesentliche Veränderungen hin, die ihn gegenüber den neuesten Entwicklungen der psychotherapeutisch orientierten Psychiatrie skeptisch stimmen.
\end{abstract}

Schlüsselwörter:Psychiatrie, Psychiatriegeschichte, Psychotherapie, Institutionskritik, Wissenschaftsideologie.

Eingeladen, anlässlich dieser Klinik-Neueröffnung über «Veränderungen in Psychiatrie und Psychotherapie» zu sprechen, möchte ich in den folgenden Ausführungen eine Art «Standortbestimmung der stationären Psychiatrie heute» vornehmen. Anlass dazu ist eine grosse organisatorischeVeränderung des weit herum geschätzten Etablissements, was a) dessen Konzept und b) dessen Bezeichnung betrifft. Solche Änderungen drücken ja immer auch den Willen und die Hoffnung einerWandlung aus und man feiert sie entsprechend, weil ja solcheWandlung eine zum «Besseren» sein soll. Dass dieses «Bessere» wohl auch rentabler sein will, muss so lange nicht angemahnt werden, als das Rentable das Bessere nicht zur Seite drängt... Es soll mit dieser Neu-Orientierung auch die Enge dieser Täler und der Gemüter erweitert werden, noch besser Anschluss zu finden an die grosse weite Welt von Wissenschaft und Medizin. Gerade hier, auf diesem Territorium, wo einst ein Knabe seinem Vater - nachdem ihm dieser die Grösse und Weite der «anderen Welt» geschildert hatte - zurief: "Vater, es wird mir eng im weiten Land - da wohn' ich lieber unter den Lawinen! (...)» und dieser dann entgegnete: «(...) Ja, wohl ists besser, Kind, die Gletscherberge im Rücken haben, als die bösen Menschen (...)»"

Veränderungen in Konzept und Form, aber auch der Inhalte, des Selbstverständnisses und derVermittlung ärztlichen Handelns sind, seitdem es die Psychiatrie als medizinische Disziplin überhaupt gibt (und das ist so lange gar nicht her - etwas 
mehr als 200 Jahre ungefähr), ein stetiger und unablässiger Kehrreim. Die Psychiatrie ist seit eh und je eine Baustelle, eine sich immer wieder wandelnde Struktur um das herum, was ihr eigentliches Kerngeschäft ist: der Behandlung «irr» gewordener Menschen, dem seelischen «Fallout» einer sich immer schneller drehenden «Normalität», jener 5-10 Prozent der Menschen, die aufgrund ihrer genetischen, konstitutionellen, ihrer entwicklungsbedingten und sozialen Voraussetzungen nicht mehr Schritt halten können mit dem strengen Diktat von Tauglichkeit, Tüchtigkeit, Unauffälligkeit und Anpassung. Derjenigen, die mangels Verfügbarkeit über herkömmliche Bewältigungformen von Leid und Freud, Angst und Unvermögen ihre jeweils eigenen und eigensinnigen «privatisierten» Reaktionsformen entwickeln und die von uns als «Störungen» und «Krankheiten» behandelt und wieder zurechtgerückt werden sollten. Sie sollen mit unserer Hilfe und wie es Sigmund Freud einmal ausdrückte, aus ihrem psychotischen und neurotischen Elend den Weg zurück ins «reale Elend» finden und dieses dann auch besser bewältigen können. Der alte, nun hinter sich gelassene Namen der Klinik möge uns wenigstens heute noch in Erinnerung bleiben : «Franziskusheim» - in Erinnerung an den heiligen Franziskus, der sich auch mit Pflanzen und Tieren verständigen konnte und dem wir insofern ähnlich sein wollen, als wir die Sprache und die Gesten jener verstehen möchten, die alle anderen nicht mehr verstehen. Allerdings wird mit dieser Umbenennung auch der bisherige Werbeslogan hinfällig: «Hast Du einen Vogel, so bring ihn ins Franziskusheim ...»

Im Laufe der erwähnten 200 Jahre hat sich im medizinischen Teilbereich der Psychiatrie sehr vieles verändert: an den grundsätzlichen Methoden, an den Begriffen, an den Behandlungsformen, an den mit diesem Spezialgebiet beauftragten Institutionen, in Lehre und Forschung, in der Diagnostik und Therapie und - wenigstens teilweise, aber auch örtlich sehr unterschiedlich - in der Rezeption von psychischen Störungen in der sogenannt normalen Umwelt. Es sollte sich zeigen, dass die Verhältnisse in der Psychiatrie (dem «Irrenwesen») zu einem oft bizarren Spiegelkabinett der jeweiligen gesellschaftlichen Dominanten und Herrschaftsverhältnisse wurden - die "Ausgrenzung der Unvernunft» (Michel Foucault), welche stets reflektiert, was die Herrschaft der jeweiligen Vernunft nicht mehr bewältigen kann.

Ich darf, ganz kursorisch und notgedrungen unvollkommen, einige wesentliche Veränderungs-Etappen aus dem Reiche der «Unvernunft» erwähnen:

Zuerst, gegen Ende des 18. Jahrhunderts, begann die Aufnahme des Irrenwesens in den grossen Schoss der Medizin. Die Betroffenen wurden nun nicht mehr als Verirrte, Besessene, asoziale Elemente usw. betrachtet, sondern als «Kranke» 
und als «Patienten» und entsprechend wurden nun nicht mehr Sozialwärter, Exorzisten oder Sittenpolizisten mit ihrer Betreuung beauftragt, sondern Ärzte und Ärztinnen, sowie zunehmend speziell dafür ausgebildetes Pflegepersonal. Wissenschaftlich wurde nach den Ursprüngen solcher nunmehr als Krankheit bezeichneten Abweichungszuständen geforscht, im 19. Jahrhundert vor allem über Neurologie und Hirnforschung - nachdem zuvor Urelemente wie Feuer, Luft, Wasser, die Körpersäfte oder der verirrte Glaube als Erklärungsmodelle gedient hatten. Die Ärzte bezeichneten sich als «Nervenärzte», arbeiteten in «Nervenheilanstalten» und behandelten «Nervosität», «Psychasthenie» und «Dekadenz».

$\mathrm{Ab}$ Mitte des 19. Jahrhunderts gesellte sich zu den organisch (heute würde man sagen den biologisch) orientierten Forschungsansätzen eine neueWissenschaft des «Seelischen», also der These, dass psychisches Geschehen und Verhalten etwas mit dem jeweils spezifisch Erlebten der Patienten zu tun habe und dass deshalb auch die Behandlung der psychisch Kranken mittels «seelischer Mittel», der Einflussnahme auf ihr Erleben also, zu verbinden sei. Dies konnte, wie z. B. in den grossen französischen Schulen von Pinel, Charcot etc. bei der nun modisch gewordenen «Hysterie» durch Hypnose oder andere suggestive Mittel geschehen, oder dann auch durch massive «Reiztherapien» wie Wasserkuren, elektrische Reizung, Ruheisolation, Dunkelkuren usw. ...

Gegen Ende des 19., anfangs des 20. Jahrhunderts wird die «Wissenschaft der Seele» durch die weitreichenden Theorien Sigmund Freuds ganz neu aufgerollt und findet über die «Zürcher Schule» (C.G. Jung, Eugen Bleuler, Adolf Meyer in den USA) einen fruchtbaren Eingang in die Psychiatrie. Das Irresein und die Verrücktheit werden als eine Sonderform des menschlichen Erlebens und seiner Konflikte betrachtet, keineswegs so fremdartig und exotisch wie man bis anhin glaubte, sondern viel ähnlicher dem, was alle Menschen in ihren Träumen und Phantasien ebenfalls erleben können. Und es wurde erkannt, dass auch bei den «Verrücktesten» noch Anteile gesunder und besonnener Ich-Strukturen bestehen, die dann auch dem Therapeutischen zugänglich sind. Die Arbeit mit den Patienten als «Partner» und nicht nur als eigentliche Krankheitsträger wurde der eigentliche Beginn der «Psychotherapie», der «talking cure».

Fast gleichzeitig entwickelte sich unter Kraepelin und Eugen Bleuler eine nomenklatorische Systematik der psychischen Krankheiten, die Ähnlichkeiten unter ganzen Gruppen psychischer Störungen definierte, aber sie auch gemäss den wahrscheinlichen Verursachungsprinzipien aufschlüsselte (von ausssen kommende «exogene» Krankheiten: wie durch Alkohol, Syphilis oder körperliche Krankheiten und die von innen kommenden «endogenen» (d. h. durch das Seelische selbst ent- 
standene) Störungen. Kraepelins Verdienst besteht darin, dass er als erster überhaupt die klinische Beobachtung der Patienten in den Mittelpunkt stellte, und daraus die Differenzierung und Klassifizierung psychischer Störungen initiierte.

So war in der ersten Hälfte des 20. Jahrhunderts das Gebiet der Psychiatrie in seinen Beschreibungs- und Systematisierungsmöglichkeiten, ja selbst in seinem psychotherapeutischen Zugang zu den Patienten zwar schon weit erschlossen, aber in den kurativen Möglichkeiten noch immer relativ hilflos. Es wurden zwar die sogenannt grossen Kuren (Insulinkur, Malaria-Fieberkur, Schlafkur, Dämmerkur, Cardiazol-Reizung, Elektrokur - sogar Hirnchirurgie) entwickelt - ohne dass damit aber das eigentliche Wesen psychischer Krankheiten grundsätzlich besser erhellt wurde.

In vielen Ländern entwickelte sich neben der institutionalisierten Psychiatrie der grossen Kliniken die «ambulante Psychiatrie» der Einzelärzte und -Ärztinnen, die sich nun nicht mehr als blosse «Nervenärzte», sondern als SpezialistInnen für Psychiatrie und Psychotherapie bezeichnen liessen. Grosse Werke zur Theorie der Psychiatrie (etwa Freuds «Fall Schreber», Eugen Bleulers «Naturgeschichte der Seele» und Karl Jaspers «Psychopathologia generalis») wurden publiziert.

Nach dem zweiten Weltkrieg begann die hohe Aera der eigentlichen «Psychopharmakologie». Es wurden - manchmal durch den Zufall ihrer Nebenwirkungen - Substanzen entdeckt, die auf das psychische Verhalten und Erleben einen kurativen Einfluss hatten, die also z. B. die psychotischen Symptome eindämmen, die Depression aufhellen oder die Erregtheit und Unruhe lindern konnten. Durch die Entwicklung der Antibiotika wurde ein Hauptlieferant der Psychiatrie, die Syphilis, fast vollständig ausgeschaltet. Als methodischer Kontrapunkt dazu wurden gleichzeitig und begünstigt von der relativen «Ruhe» (und der gesellschaftlichen Langeweile) der prosperierenden Nachkriegsjahre neue institutionelle Modelle mit erhöhter Partizipation der Patienten entwickelt, die «therapeutic community» etwa, sowie die ambitiösen Modelle einer intensiven Psychose-Psychotherapie.

Zu dieser Zeit (1964 - also vor fast 45 Jahren, inzwischen 2/3 seines Lebens) betritt auch der hier Schreibende das «grosse Floss der Psychiatrie» - wie es einer seiner Lehrer nannte - «Floss» deshalb, weil wir nie vergessen sollten, dass sich diese «Wissenschaft» letztlich immer auf wackligem Untergrund bewegt.

Parallel und zeitlich fast synchron zu den gesellschaftlichen Turbulenzen der 60er-Jahre (den «68ern») entstand eine in den westlichen Ländern sich rasch ausbreitende Psychiatrie-Kritik. Die Psychiater seien nichts anderes als die Kustoden der gesellschaftlichen Ausgrenzung, ihre Institutionen und Methoden seien gleichzeitig Verwalter und Mitverursacher des psychischen Elends, einäugig auf das 
«Kranke» konzentriert und blind für die sozialen und gesellschaftlichen Faktoren psychischen Leidens. Es entstand die «Anti-Psychiatrie» mit den theoretischen Werken von Laing, Cooper, Goffmann, Szasz etc. und einer politisch-psychiatrischen Bewegung in Italien unter Basaglia, die erwirkte, dass 1974 per Gesetz die Schliessung aller psychiatrischen Krankenhäuser dekretiert wurde. In der Schweiz, unter den Lawinen eben, wo wir etwas langsamer und konfliktscheuer sind, wurde die «Sozialpsychiatrie» als Tochtergesellschaft der grossen Seelenholding entwickelt, anfänglich als konkurrierender Wettbewerbspartner zur klassischen Psychiatrie, heute integriert in dieselbe und - wie in fast allen Ländern Westeuropas - zuständig für die territoriale Erfassung und Betreuung der langfristig von der Psychiatrie abhängigen Patienten und Patientinnen.

Wie einst im grossen Zirkus «Barnum \& Bailey» gibt es heute auch im Psychiatriezirkus vieler Länder drei Arenen:

, die psychiatrischen Kliniken - meistens abgespeckt, modernisiert, zum grossen Teil offen, geschlechtlich durchmischt und, auch wo sie sich «privat» nennen, mit öffentlichem Auftrag (wie hier: «Vertragskliniken für die Kantone Uri, Schwyz und Zug»);

, die öffentlichen, oft regional organisierten Institutionen der «Sozialpsychiatrie» mit ihren Ambulatorien/Polikliniken, den Kriseninterventionszentren, den Tages- und Nachtkliniken, den therapeutischen Wohngemeinschaften;

, die psychiatrische Privatpraxis, in der Schweiz (im Gegensatz zu vielen anderen Ländern) noch immer eine Bastion der freigewählten und offenen Begegnung zwischen Patient und Arzt - hier wird, als intimes Kammerspiel sozusagen, noch am häufigsten die eigentliche Psychotherapie betrieben, häufig auch unter Delegierung an fachkundige und kompetente PsychologInnen/ PsychotherapeutInnen.

Soweit zur Organisation der Psychiatrie bis zum heutigen Zeitpunkt. Was aber hat sich in ihrem «Inneren» sozusagen inzwischen weiter verändert? Und wo stehen wir heute?

Fast unbemerkt von der breiten Öffentlichkeit, ja auch meistens ohne Mitwissen der direkt Betroffenen, der Patienten und Patientinnen nämlich, sind Veränderungen im Gange von solcher Tragweite, dass man fast von Mutationen in statu nascendi sprechen möchte.

Wie schon eh und je spiegelt sich in der Psychiatrie - wenn auch oft wie in jenen Zerrspiegeln, die uns manchmal zu dünn, zu dick, zu kopflastig oder zu dünnbeinig erscheinen lassen - die gesellschaftlicheWirklichkeit wieder, heute mit dem Begriff «Globalisierung» und «Ökonomisierung» versehen. Galten früher in der 
Medizin vor allem die Forderungen nachZweckmässigkeit undWirksamkeit ärztlichen Handelns, so kommt heute ganz prägend das Postulat der «Wirtschaftlichkeit» dazu. Allein dies führt in der praktischen Arbeit zu ganz erheblichen Veränderungen. Dazu kommt noch das für die Psychiatrie klassische Paradoxon, dass die nach objektiven Kriterien ausgerichtete therapeutische Arbeit mit einem ganz extrem gestalteten Subjektivismus seitens der Patienten konfrontiert wird. Denn die als «psychisch krank» bezeichneten Phänomene zeichnen sich ja gerade dadurch aus, dass sie sich den allgemein gültigen Regeln des Erlebens, des Handelns und derVorstellung über das den meisten Menschen Gemeinsame entziehen. Wenn wir uns als Ärzte und Ärztinnen, als PsychotherapeutInnen mit unseren Patienten identifizieren wollen (und nur dieses ermöglich überhaupt die «therapeutische Beziehung»), ziehen sie uns auf die Seite ihrer Subjektivität und weg von den heiligen Regeln der normativen Objektivität. Dieser «therapeutische Spagat» überfordert viele meiner beruflichen Artgenossen, nicht zuletzt, weil die unserer Arbeit oft beigesellte «Ohnmacht» eher zu emsiger Betriebsamkeit als zu vertiefter Kontemplation, eher zu einem noch stärkeren Bedürfnis nach Objektivität als nach hilfloserVerlegenheit drängt. Werden objektive Diagnosen, Statistiken, Kosten, Bettenbelegung und Therapiedauer oder -Frequenzen zur Richtschnur der Qualitätskontrolle psychiatrischen Handelns, so führt dies zu ganz massiven Veränderungen der therapeutischen Mentalität und damit unweigerlich auch der psychiatrisch/psychotherapeutischen Kultur.

Ich möchte von diesen Veränderungen nur drei, der mir am wichtigsten erscheinenden benennen ( selbst auf die Gefahr hin, dass man einem alten Player nach 45 Jahren psychiatrischem Aktivdienst unterstellen könnte, er blicke wie so viele Senioren mit Misstrauen und Argwohn auf die neuen Entwicklungen hin, die sich ihm langsam zu entziehen drohen ).

\section{Die neue Nomenklatura}

In den letzten ca. fünfzehn Jahren wurde versucht, die psychiatrischen Krankheiten weltweit zu erfassen, zu vergleichen und verbindlich zu codieren. Daraus entstanden zwei korrespondierende Systeme, die heute nicht nur allgemeine Gültigkeit, sondern auch wegleitenden Einfluss auf die psychiatrische Kommunikation angenommen haben. Es sind dies das ICD 10 («International Classification of Mental and Behavioural Disorders» in der 10. Auflage), sowie das DSM IV («Diagnostic Manual of Mental Disorders» in der 4. Auflage). Wie Sie wissen, sind dies relativ dicke Bücher, in denen alle psychischen Störungen nach Gruppen, Entstehungsursachen und Erscheinungsbildern erfasst und mit jeweiligen Diagnosenummern oder Codes versehen sind. Die Nützlichkeit dieser Systematik 
ist für die Verbindlichkeit der Diagnosestellung und für die wissenschaftlichen Möglichkeiten einer disziplinierten, nunmehr globalisierten Kommunikation unbestritten. Sie hat nur einen, allerdings ganz entscheidenden Nachteil: dass nämlich die genaue Beschreibung und Klassifizierung eines zutiefst rätselhaften Phänomens, wie dasjenige der seelischen Veränderungen, nicht identisch ist mit der komplexen Tragweite eben dieser Phänomene. Es wäre ungefähr so, als könnten sie die Turbulenzen und Veränderungen in Ihren partnerschaftlichen Beziehungen jeweils in irgendwelchen Kompendien nachschlagen, um dann daraus eine genaue Diagnose des Beziehungszustandes, der daran beteiligten Gefühle und letztlich sogar die eventuellen Richtlinien für Ihr Handeln daraus ableiten. Für die Psychiatrie, aber auch für die Psychotherapie (für welche es inzwischen eine ähnliche Systematik, das OPD «Operationalisierte Psychodynamische Diagnostik», in der 3. Auflage gibt) ist daraus eine Verlagerung des klinischen Denkens und Handelns ganz einseitig in Richtung Diagnostik entstanden, was an sich zwar keineswegs unwichtig, aber für die Arbeit mit Patienten tendenziell verhängnisvoll ist. Will ich vor allem wissen, was für eine Krankheit der Patient hat und wie ich sie zu codieren habe, so wird dies zwar der statistisch relevanten Forderung nach Objektivierung gerecht, nicht aber und noch keinesfalls dem spezifischen Erleben, ja der Einmaligkeit desselben für eben diesen Patienten. Und meine eigenen psychischen Reaktionen auf das Rätselhafte, was diese «Störung» in mir in Bewegung versetzt - und was für jede Psychotherapie unerlässlich ist - werden kurzerhand im gekröpften Anflug auf die Diagnose eliminiert. Aber auch auf die Ausbildung der jungen Kollegen und Kolleginnen hat diese tendenzielle Hegemonie der Diagnostik einen nicht zu unterschätzenden, aus meiner Sicht negativen Einfluss. Das Verweilen und Hinsehen, das nur sehr langsam und in schwierigen Prozessen erlangbare hoch-individuelle Wissen über den jeweiligen Patienten - zunächst ganz ungeachtet seiner Diagnose - droht unterzugehen und nimmt der Faszination eben dieser Arbeit die Aura der unerlässlichen Subjektivität. An den erwähnten Veränderungen ändert sich auch dann nichts, wenn man sie mit dem netten Bastardenbegriff «bio-psycho-sozial» versieht.

Der Knabe Tell würde heute singen :

Mit dem Fragebogen

durch Praxis und Spital

kommt der Arzt gezogen

und codiert den Fall ...2 


\section{Die biologistische Wiedergeburt und das Milliardenbusiness}

Durch die rasanten technologischen und wissenschaftlichen Entwicklungen auf dem Gebiete der Neurobiologie lassen sich in hochdifferenzierten bildgebenden Verfahren psychische Vorgänge im menschlichen Gehirn abbilden und untersuchen. So lässt sich den Träumen, den Ängsten, den Zwängen, ja selbst der Phantasieaktivität nachspüren und pathologische Veränderungen der Psyche werden korreliert mit ihrem anatomisch-physiologischen Substrat. Ohne Zweifel sind dies epochale Fortschritte. Mit Zweifel aber kann man den daraus hervorgehenden Trugschluss feststellen, wenn dann die Illusion entsteht, man habe nun tendenziell sämtliche Formen des menschlichen Erlebens im Griff. Gewiss, gab es schon immer Versuche die menschlichen Regungen, wie z.B. Liebe, Trauer, Hoffnung - etwa in Poesie oder bildender Kunst - «abzubilden», doch nie wurde daraus geschlossen, man wisse jetzt genau und eindeutig, ja sogar «objektiviert», wie sich dieses bei den Menschen oder insbesondere beim spezifischen Menschen Brunner oder Müller dann auch wirklich verhalte. Die neuen neurobiologischen Erkenntnisse sind wertvoll als Ergänzung zu den immer nur tastenden und hypothetischen Vorstellungen über das, was sich im Menschen seelisch abspielt. Sie sind aber nicht, können nicht sein, die eigentliche Übersetzung des Geheimnisses von bewusstem oder unbewusstem Erleben. Es liegt auf der Hand, dass wir mit solchen Argumenten bereits in den Bereich von «Glaubensbekenntnissen» vorstossen, d.h. wir kommen plötzlich in die seltsame Lage, dass wir die «Begegnung» mit den Patienten, unsere Beziehung zu ihnen auf eine Art «verteidigen» müssen, als handle es sich um eine romantische Blauäugigkeit gegenüber den modernen «hard facts» der Naturwissenschaft. Zwar waren die PsychiaterInnen auch immer der Meinung sie betrieben Naturwissenschaft, indem sie die geheimnisvolle Natur der menschlichen Seele erschliessen wollten, aber sie suchten den Pfad dazu in der Begegnung mit den Patienten und in der «therapeutischen Beziehung», die über das darin stattfindende gegenseitige Erleben, Einblicke und Austausch ermöglichen sollte. Es ist dies, neben der medikamentösen Behandlung, das wesentliche Element der psychiatrisch/psychotherapeutischen Bemühungen. Ohne Zweifel wird heute das vormalige Gleichgewicht dieser methodischen Ausgangspunkte tendenziell verschoben in Richtung der biologistischen Betrachtungsweise, was in Forschung und Lehre, in Ausbildung, Betreuung und Begegnung mit den Patienten von erheblicher Tragweite sein wird. Ich bin über diese Entwicklungen - nicht nur aus nostalgischen Altersgründen - äusserst skeptisch, weil ich glaube, dass damit ein wesentlicher Aspekt der Faszination und der «challenges» in unserem Beruf verloren gehen wird. 
Die Macht und Wucht dieser biologistischen Renaissance beruht zwar, so ist zu hoffen, auf ihrem Wahrheitsgehalt, sie hat aber auch noch einen mächtigen Schwager. Es ist dies der pharmazeutisch-industrielle Komplex - dessen Firmen die gewaltigen Kosten der neurobiologischen Forschung zu einem grossen Teil finanzieren, Ganz selbstlos sind aber diese Investitionen nicht. Denn über die Neurobiologie und Neurochemie eröffnen sich Märkte von gigantischen Dimensionen: nicht nur in erster Linie über die Hightech-Instrumente und Apparate, die für die Forschung unerlässlich sind, sondern über die Entwicklung und Vermarktung der Psychopharmaka - ein Milliardengeschäft, wie man weiss. Dazu kommt noch, dass sich die Tentakel des Pharma-Kolosses sogar in die erwähnte Nomenklatura hinein wühlen - es besteht die begründeteVermutung, dass bestimmte «neuere Syndrome» (wie die Panikstörung, die soziale Phobie, das Attention-deficite Syndrome und andere) erst kreiert und definiert wurden, als die dafür einzusetzenden Medikamente bereits in der Pipeline schlummerten.

So gibt es heute Medikamente gegen Depressionen, gegen psychotische Zustände, gegen psychische Labilität und selbst gegen Altersdemenz, die tatsächlich Hilfe und Entlastung für die Betroffenen aber auch für die Mühsal der Ärzte und des Pflegepersonals, sowie der Angehörigen bewirken und die in einer modernen Psychiatrie nicht mehr wegzudenken sind. Nur befreien uns auch diese nicht vor dem bereits erwähnten Dilemma: nämlich den Patienten in seinen innersten Regungen, seinen Konflikten und seinem ver-rückten Erleben wirklich zu verstehen. Ja, kann man fragen, ist denn solches überhaupt noch nötig, wenn wir diese Patienten auf andere Weise heilen können? Leider kann von «Heilung» nur in den seltensten Fällen gesprochen werden. Die gesuchte Linderung aber, wird nicht nur mit manchmal erheblichen Nebenwirkungen (Gewichtszunahme, Libidoverlust, hormonale Nebeneffekte, metabolisches Syndrom etc.) erkauft, nein, die viel grössere Nebenwirkung besteht wohl darin, dass sich in den Köpfen der Betreuer bei einem äusserst komplexen Sachverhalt eine Kurzschlussmentalität einschleicht, die zwar das jeweilige Verhalten der Patienten beeinflussen kann, nicht aber wiederum sein damit verbundenes Erleben, das in ihm einmalig, unverwechselbar, biographisch, sozial, kulturell und existenziell geheimnisvoll eingraviert ist. Die «Todeslandschaften der Seele», wie G. Benedetti die rätselhafte Innenwelt psychotischer Patienten nennt, wird nicht besser erschlossen, wenn darüber noch so elegante und kühne Viadukte errichtet werden. 


\section{Die psychotherapeutische Erosion}

Unter diesen massiven Veränderungen in der Welt der vormaligen SeelenMedizin durch die Nomenklatura, die biologistische Renaissance und die mächtige Pharmaindustrie wird die ehemalige Kernzone psychiatrischen Denkens und Handelns, das Konzept der Psychotherapie nämlich, tendenziell und faktisch immer mehr an den Rand gedrängt. Psychotherapie bedeutet ja die Erforschung und Bearbeitung der «inneren Welt» der Patienten sowie der Versuch, über ganz spezifische (wenn auch unter sich sehr unterschiedlichen) Beziehungsentwicklungen im Dialog, der Gruppe oder dem Milieu, dem veränderten und störenden Erleben des Patienten auf die Spur zu kommen und es über einen Erkenntnis- und Gefühlsprozess zu verändern. Diesem liegt meistens dieVermutung zugrunde, dass in den psychischen Störungen immer auch eine «psychogene», d. h. im Seelischen selbst entstandene, konfliktbeladene Unfreiheit vorliege. Solches ist nicht identisch mit dem «ärztlichen Gespräch», der Nettigkeit und Freundlichkeit den Patienten gegenüber, die ja - so würde man meinen-in allen Branchen der Medizin eine Minimalvoraussetzung sind. Gemeint ist ein sich vortastendes Einlassen in die spezifische und jeweils einmalige Erlebniswelt des Patienten, sowie derVersuch über die Gestaltung einer ganz spezifischen «therapeutischen Beziehung», dieAusdrucks- und Empfindungsmöglichkeiten sowohl des Patienten, als auch des/der TherapeutIn in neue, weniger schädliche Bahnen zu lenken. Kein leichtes Unterfangen fürwahr! Oft mühselig, langweilig und hartnäckig und dazu noch ohne sichere Garantien für den Ausgang. Sie erfordert von den TherapeutInnen eine lange Ausbildung, Erfahrung/Selbsterfahrung und eine Resistenz gegen schnelle und oberflächliche Lösungen, die vielleicht die Symptome günstig beeinflussen, nicht aber dasjenige für das die Symptome (die «Zeichen») stehen, nämlich die komplexen, verschlungenen und oft verknoteten Strukturen der seelischen Innenwelt.

Ja, gibt es denn die Seele, das Seelische überhaupt noch, wenn sie identisch sind mit dem Hirn und seinen Funktionen? Grundsätzlich ist diese Frage berechtigt. Wenn man sie verneint, dann können wir sie (die Seele) wieder ganz den Theologen und den Philosophen überlassen und uns hinfort vorwiegend der Klassifizierung/Codierung/Diagnostizierung und Pharmakologisierung widmen. Das «freundliche Gespräch» kann auch so noch erhalten bleiben. Und wird ja in den Begleitzetteln der Medikamente auch stets empfohlen.

Damit aber, so befürchte ich, würde der Psychiatrie, so wie sie sich über zwei Jahrhunderte entwickelt hat, eine ihrer wesentlichen Tragsäulen genommen Die Arbeit mit den Patienten würde immer mehr formalisiert und operationalisiert, die Patienten würden zwar statistisch besser erfasst, in ihrem Erleben aber sich 
selbst überlassen, die Tätigkeit in Klinik und Praxis würde eintöniger und kälter (Entschuldigung! cooler muss man heute sagen) und wir würden in der unablässigen Erforschung des «Irrsinns», des «Wahns», der «Zwänge» und «Ängste» immer weiter vom Subjekt weg-gedriftet. Wir würden von den Viadukten des Machbaren auf die Wildnisse und Dschungelpfade der seelischen Irrwege herabblicken - nicht ganz teilnahmslos zwar, aber ohne wirkliche Teilnahme und Beziehung dazu. Ob das gut herauskommt? - Man darf zweifeln.

\section{Anmerkungen}

$1 \quad$ Friedrich Schiller: Wilhelm Tell, 3.3.

$2 \quad$ Friedrich Schiller: Wilhelm Tell, 3.1. 